\title{
Family physician workloads and access to care in Winnipeg: 1991 to 2001
}

\author{
Diane E. Watson, Alan Katz, Robert J. Reid, Bogdan Bogdanovic, Noralou Roos, Petra Heppner
}

Abstract

Background: Current perceptions of family physician (FP) shortages in Canada have prompted policies to expand medical schools. Our objective was to assess how FP supply, workloads and access to care have changed over the past decade.

Methods: We used an anonymized physician and population registry and administrative health service data from Winnipeg for the period 1991/92 to 2000/01. We calculated the following measures of supply and workload: ratios of FPs to population, of population to FPs and of FP full-time equivalents (FTEs) to population, as well as FP activity ratios (sum of FTEs/number of FPs), annual number of visits per FP and visits per FP per full-time day of work. Trends in FP remuneration were analyzed by age and sex. We also measured standardized visit rates and stratified the analysis by populations deemed at risk of needing FP services.

Results: In 2000/01 FPs between 30 and 49 years of age $(64 \%$ of the workforce) provided $20 \%$ fewer visits per year than their same-age peers did 10 years previously. Conversely, FPs 60 to 69 years of age $(11 \%$ of the workforce) provided 33\% more visits per year than the corresponding group a decade earlier. On a per capita basis, the number of FPs declined by $5 \%$, from 97 per 100000 population in 1991/92 to 92 per 100000 population in 2000/01, which paralleled changes in national estimates of FP supply. Per capita visit rates among Winnipeg citizens (3.5 per year in 2000/01) and average workloads among FPs (4193 visits per year in 2000/01) were stable over the decade.

Interpretation: Despite relative homeostasis in aggregate FP supply and use, there have been substantial temporal shifts in the volume of services provided by FPs of different age groups. Younger FPs are providing many fewer visits and older FPs are providing many more visits than their same-age predecessors did 10 years ago, a finding that was independent of physician sex. Given these data, the perpetual focus of policy-makers and care providers on increasing numbers of FPs will not help in diagnosing or treating issues of supply, workloads and access to care.

CMAJ 2004;171(4):339-42

T $2002,80 \%$ of Canadians believed that there was a shortage of "family doctors," and $97 \%$ of these people believed that the shortage was serious. ${ }^{1}$ These perceptions are amplified within the physician workforce itself, with $93 \%$ of physicians surveyed in the same year believing that shortages among their ranks were widespread. ${ }^{2}$ Para- doxically, current perceptions of shortages and suggestions to fast-track increases in supply $y^{3,4}$ come close on the heels of widespread perceptions of surpluses, at least in urban centres, and reductions in medical school enrolment only 10 years ago. For example, in 1993, a Canadian Medical Association survey found that half of the doctors in the country believed enrolment in medical schools should be reduced. ${ }^{5}$ Such relatively quick shifts from perceptions of surpluses to ones of shortages are at odds with recent national data indicating that the per capita number of FPs has remained relatively stable over the last decade. ${ }^{6,7}$

Unfortunately, temporal patterns of FP workloads over the past decade have not been documented in national supply-side analyses. ${ }^{6.8}$ Furthermore, analyses have not been conducted to understand temporal patterns in the relation between FP age or sex and workloads. Yet this type of information is vital to understanding (and projecting) the impact of demographic shifts in the workforce ${ }^{8}$ on current (and future) supply of services. When the supply of FPs is inadequate, the workloads of both FPs and specialists could be affected and access to care compromised. There is evidence that FPs work long hours, ${ }^{9}$ that many are unhappy with their workloads ${ }^{9,10}$ and that those who report heavy workloads are more likely than others to stop accepting new patients.

The purposes of this study were to evaluate whether FP workloads, on average or for certain cohorts of practitioners, have changed over a period of relatively stable FP-topopulation ratios; to simultaneously examine the population's use of FPs; and to consider the potential impact of any change in workloads or service utilization on perceptions of adequacy of FP supply and access to care. This population-based study, which formed part of a larger project, ${ }^{11}$ was based on data from Winnipeg, a city (like others in Canada) where FPs report unhappiness with workloads, where citizens express frustration regarding access to FPs and where journalists document widely held views that many FP practices are "restricted" in accepting new patients. ${ }^{12}$ We hypothesized that average workloads increased, that population rates of use declined, and that age- and sexspecific workloads remained constant.

\section{Methods}

Our analyses relied on anonymized physician and population registry data and health services data for fiscal periods (ending 
Mar. 31) $1991 / 92$ to $2000 / 01$ from the Population Health Research Data Repository at the Manitoba Centre for Health Policy. ${ }^{13}$ The data accounted for all salaried FPs (7\% of the workforce in 2000/01) and those who billed on a fee-for-service basis (93\% of the workforce). The proportion of FPs receiving salary

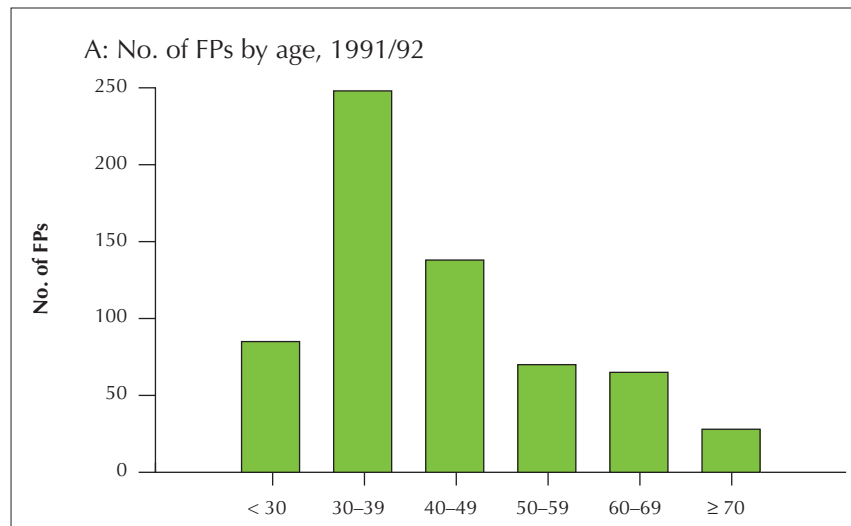

B: No. of FPs by age, 2000/01

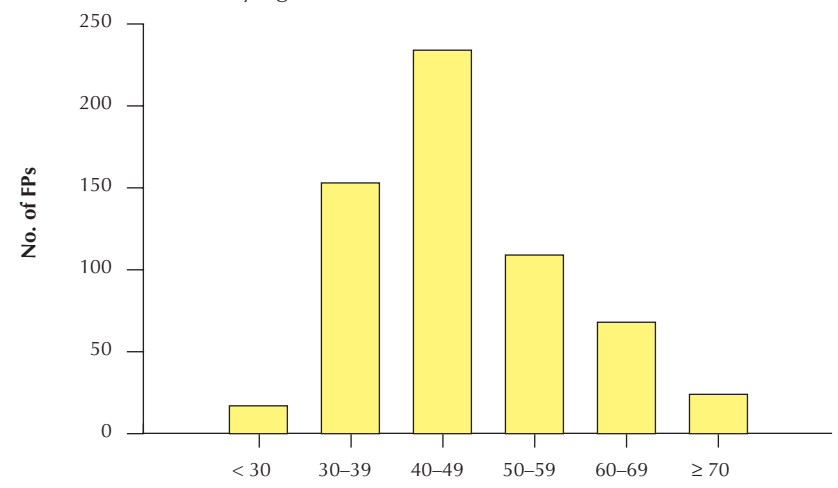

C: Mean annual visits per FP, by age

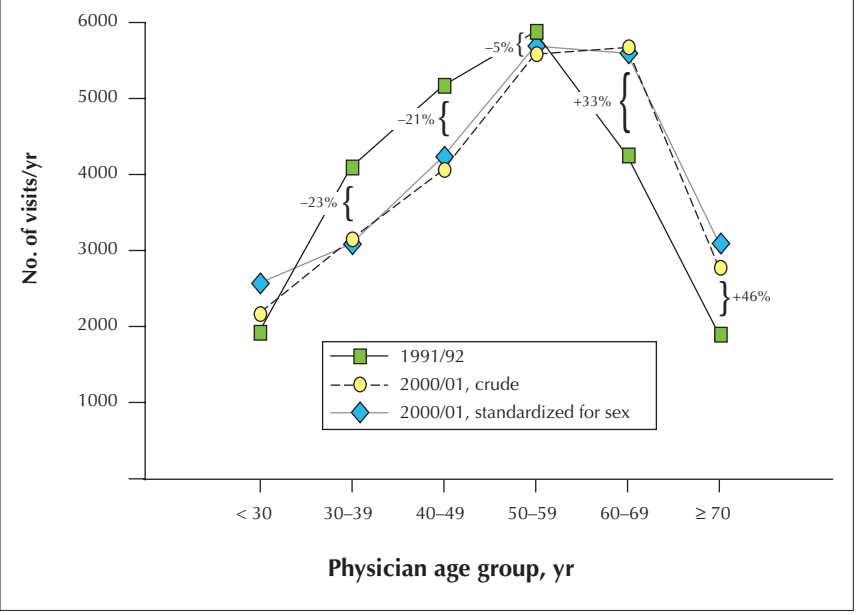

Fig. 1: Number of Winnipeg family physicians (FPs) by 10-year age group in 1991/92 (A) and 2000/01 (B), and mean annual number of visits provided per FPs by physician age, 1991/92 and 2000/01 (C). Fig. 1C also presents the percent change in number of visits for each physician age group from 1991/92 to 2000/01 (these calculations are based on the crude data for 2000/01). did not change substantively over the study period. For each year, we calculated ratios of FPs to population, of population to FPs and of FP full-time equivalents (FTEs) to population, where FTEs are workload values that rank physicians relative to their peers on the basis of practice income. Temporal trends in workloads were assessed on the basis of activity ratios (i.e., sum of FTEs divided by number of practising FPs), visits per FP per year and visits per FP per full-time day of work. Changes over time in the relation between age and workload were standardized for sex to isolate the effect of age and to account for temporal increases in younger female FPs. To better understand whether shifts in workloads were related to changes in the magnitude of remuneration, we calculated price-adjusted billings (i.e., constant dollars).

We measured trends in crude and standardized visit rates for Winnipeg citizens using the direct method, with 1991/92 as the standard. Use measures were also calculated for populations with higher-than-average needs for primary care services, which can be identified from administrative data with validated methodology (i.e., people with diabetes, hypertension or a mental health disorder, as well as those 85 years of age or older). A visit was defined as any face-to-face contact in an office, private residence, nursing home or emergency department. Visits with hospital inpatients and for prenatal care were excluded. ${ }^{11}$

\section{Results}

Between 1991/92 and 2000/01, the number of FPs in Winnipeg declined from 634 to 605 (decrease of $5 \%$ ), while the size of the population remained essentially constant (653 452 and 654930 respectively). The FP-topopulation ratio peaked between 1993/94 and 1995/96 and then declined by $8 \%$ by $2000 / 01$. Over the 10 years, the number of FP FTEs declined by $3 \%$ (from 470 to 454 ) and the FTE-to-population ratio declined by $4 \%$ (72 to 69 per 100000 population). The proportion of FPs who worked part-time remained stable at about $57 \%$ throughout the study period. The average number of visits provided per FP each year was also stable (4198 in 1991/92 and 4193 in $2000 / 01$ ), as was the average number of visits provided per FP on a full-time day of work (27.9 and 27.8 visits respectively). (See Appendix 1 at www.cmaj.ca/cgi/content/full /171/4/339/DC1 for the complete data.) Standardized per capita visit rates declined by $3 \%$ to a level of 3.5 in 2000/01, and visit rates for populations with higher-thanaverage needs for primary care services remained relatively stable (analysis not shown). ${ }^{11}$

Over the 10-year period, the average age of FPs increased by 3.8 years (from 42.6 to 46.4 years), which appears primarily attributable to the aging of "baby boomer" physicians (Figs. 1A and 1B). Between 1991/92 and 2000/01, the proportion of female FPs increased slightly, from $29 \%$ to $31 \%$. The relation between FP age and workload took an inverted $\mathrm{U}$ shape, but the nature of this association changed over time (Fig. 1C). In 2000/01, FPs between 30 and 49 years provided approximately 20\% fewer visits each year, on average, than their same-age peers did in 1991/92. FPs 50 to 59 years of age provided $5 \%$ fewer visits. Conversely, FPs 60 to 69 years of age provided 33\% 
more visits each year, and FPs 70 years of age or older provided $46 \%$ more visits than their same-age peers in 1991/92. Similar trends were evident for activity ratios, total billings from patient visits and price-adjusted billings from these encounter data (data not shown). Shifts in the relation between age and workload within the workforce were not attributable to shifts in male to female ratios (see sex-standardized data for 2000/01 in Fig. 1C).

Workloads appeared relatively stable within age cohorts across time. For example, FPs 30 to 39 years of age in $1991 / 92$ were 40 to 49 years old in 2000/01. At the beginning and end of the decade, this cohort delivered roughly the same number of visits per year (about 4100). The annual number of visits provided by FPs 40 to 49 years of age in 1991/92, who were 50 to 59 years of age in 2000/01, increased slightly over the decade (from almost 5200 in $1991 / 92$ to roughly 5600 in 2000/01). Lastly, the annual number of visits provided by FPs 50 to 59 years of age in 1991/92, who were 60 to 69 years of age in 2000/01, declined slightly over the decade (from almost 5800 in 1991/92 to roughly 5600 in 2000/01) (Fig. 1C).

\section{Interpretation}

Contrary to our expectations, measures of per capita supply of FPs, average FP workloads and per capita use were stable across the 10-year period. Although others have also documented temporal homeostasis in aggregate supply and use of FPs in British Columbia ${ }^{14}$ the most intriguing finding of our study is that, despite this stability, there were substantive shifts in age-related workloads. By 2000/01, FPs between 30 and 49 years (the baby boomer generation, constituting $64 \%$ of the workforce) provided approximately $20 \%$ fewer visits per year to their patients than did their same-age peers 10 years previously. Quite the opposite pattern was detected among soon-to-retire FPs $(11 \%$ of the workforce), who had very high workloads throughout their fifties and, unlike their predecessors in this age group, did not appreciably slow their workloads after age 60. At the end of the decade that we analyzed, the workloads of older FPs were more than $30 \%$ higher than their same-age predecessors.

Why, then, the dramatic shift in public perceptions regarding adequacy of FP supply and access to FPs, when the rates of use were actually stable? The answer may lie in the relatively new phenomenon of family practices being "closed" or "restricted" in accepting new patients. In research conducted by others, roughly $70 \%$ of younger physicians reported that their workloads were heavier than they would like, 9 and FPs who felt that their workloads were too heavy were more likely than others to restrict their practices. ${ }^{6}$ Among older FPs, high and persisting workloads may be at or above the level they desire before retirement, such that these doctors may also restrict their practices. Awareness of such restrictions, independent of changes in supply or use patterns, may unduly influence public perception of the adequacy of FP supply.

However, it is important to note that baby boomer FPs are not working less than previously; over time, the volume of visits they provide each year (and real incomes) has been relatively stable or has increased slightly. However, the volume of visits to their practices in 2000/01 was substantially less than that of their same-age predecessors. The difference in workloads between older and younger FPs has become accentuated over time, which may explain perceptions that younger cohorts work differently from their older colleagues and that soon-to-retire FPs have difficulty finding younger replacements willing to assume the workloads associated with their practices.

An important limitation of our study is that we do not know how workload volumes are related to time spent engaging in work. However, the trends reported here are remarkably similar to temporal analysis of national survey data regarding the number of hours FPs of different ages spent providing direct patient care ${ }^{15}$ (Lynda Buske, Canadian Medical Association: personal communication, 2003). We were also unable to assess the relation among FP workloads, quality of care and patient outcomes.

Irrespective of the forces altering the relation between FP age and workloads, such change has been rapid in Winnipeg. If this is the case elsewhere in Canada, human resource planners must ensure that surveillance efforts include information about workload trends by physician age. Given the long lags between recent expansions in medical schools and changes in supply, this analysis indicates that current perceptions of FP availability are likely to get worse before they get better. Pending retirements by highly productive physicians could have profound implications for the availability of primary and secondary care, particularly if FP workloads shift to specialists. Strategies to enhance the efficiency of FP services will be required. Until we determine the extent and nature of change in other jurisdictions, understand the dynamics that drive such change and assess the implication of these trends on quality of care and population health outcomes, our perpetual focus on numbers of physicians is not going to help in diagnosing or treating issues of supply, workloads and access to care.

This article has been peer reviewed.

From the Manitoba Centre for Health Policy, Department of Community Health Sciences (Watson, Katz, Bogdanovic, Roos) and the Department of Family Medicine (Katz), University of Manitoba, Winnipeg, Man.; the Centre for Health Services and Policy Research, University of British Columbia, Vancouver, BC (Watson, Reid, Heppner); and the Center for Health Studies, Group Health Cooperative, Seattle, Wash. (Reid)

Competing interests: None declared.

Contributors: Diane Watson, Alan Katz, Rob Reid and Noralou Roos contributed substantially to the conception and design of the study. Diane Watson, Alan Katz Bogdan Bogdanovic and Petra Heppner contributed substantially to the analysis and interpretation of the data, and Rob Reid and Noralou Roos contributed substantially to the interpretation of the data. Diane Watson drafted the original version of the article and was responsible for all subsequent drafts after receipt of feedback from all contributors. Diane Watson, Alan Katz, Rob Reid and Noralou Roos revised the article critically for important intellectual content, and Bogdan Bogdanovic and Petra Heppner revised the article critically to ensure accurate reflection of the data. All authors approved the final version to be published. 
Acknowledgements: This work was supported as part of the project "Supply, Availability and Use of Family Physicians in Winnipeg, 1991/92-2000/01," one of several projects undertaken each year by the Manitoba Centre for Health Policy under contract to Manitoba Health. The results and conclusions are those of the authors, and no official endorsement by Manitoba Health is intended or should be inferred.

\section{References}

1. College of Family Physicians of Canada. 4.5 million Canadians not able to get a family physician [news release]. Toronto; The College; 2002 Nov 7. Available: www.cfpc.ca/English/cfpc/communications/news\%20releases/2002\% $2011 \% 2007 /$ default.asp?s=1 (accessed 2004 June 22)

2. Health care in Canada survey 2002. Toronto: Pollara; 2002. Available: www.pollara.ca/new/Fs_lib.html (accessed 2004 Apr 24). Click on "Pollara reports" to access downloadable file.

3. Expert Panel on Health Professional Human Resources, Ministry of Health and Long-Term Care. Shaping Ontario's physician workforce: building Ontario's capacity to plan, educate, recruit and retain physicians to meet bealth needs. Toronto: Ministry of Health and Long-Term Care; 2001.

4. Standing Senate Committee on Social Affairs, Science and Technology. The bealth of Canadians - the federal role. Final report. Vol. 6. Recommendations for reform. Ottawa: Senate of Canada; 2002.

5. Bueckert D. Foreign MDs limit backed in survey: Canadian doctors feel job crunch. Winnipeg Free Press 1993 June 22; Sect A:2.

6. Chan B. From perceived surplus to perceived shortage: What happened to Canada's physician workforce in the 1990s? Ottawa: Canadian Institute of Health Information; 2002.
7. Watson D, Roos N, Katz A, Bogdanovic B. Is a $5 \%$ decline in physician supply significant? [letter]. Can Fam Physician 2003;49:566-7.

8. Canadian Institute for Health Information. The practicing physician community in Canada, 1989/99 to 1998/99. Ottawa: The Institute; 2001.

9. College of Family Physicians of Canada. Updated data release of the 2001 National Family Physician Workforce Survey. Toronto (ON); The College; 2002. Available: www.cfpc.ca/local/files/Programs/Janus\%20project/ NPWS2001_Final_Data_Release_rev_en.pdf (accessed on 2004 July 1).

10. Sullivan P, Buske L. Results from CMA's huge 1998 physician survey point to a dispirited profession. CMA7 1998;159(5):525-8.

11. Watson DE, Bogdanovic B, Heppner P, Katz A, Reid R, Roos NP. Supply, availability and use of family physicians in Winnipeg, 1991/92-2000/01. Winnipeg: Manitoba Centre for Health Policy; 2003.

12. Square D. Manitoba increases med school enrolment in attempt to fight doctor deficit. CMA7 2001;164(3):395.

13. Roos LL, Mustard CA, Nicol JP, McLerran DF, Malenka DJ, Young TK, et al. Registries and administrative data: organization and accuracy. Med Care 1993;31:201-12.

14. Barer ML, Evans RG, McGrail KM, Green B, Hertzman C, Sheps SB. Beneath the calm surface: the changing face of physician-service use in British Columbia, 1985/86 versus 1996/97. CMA7 2004;170(5):803-7.

15. Buske L. Younger physicians providing less direct patient care. CMAf 2004; 170(8): 1217

Correspondence to: Dr. Diane E. Watson, Centre for Health Services and Policy Research, University of British Columbia, 2194 Health Sciences Mall, 4th Floor, Vancouver BC V6T 1Z3; fax 604 222-8635; diane.watson@chspr.ubc.ca

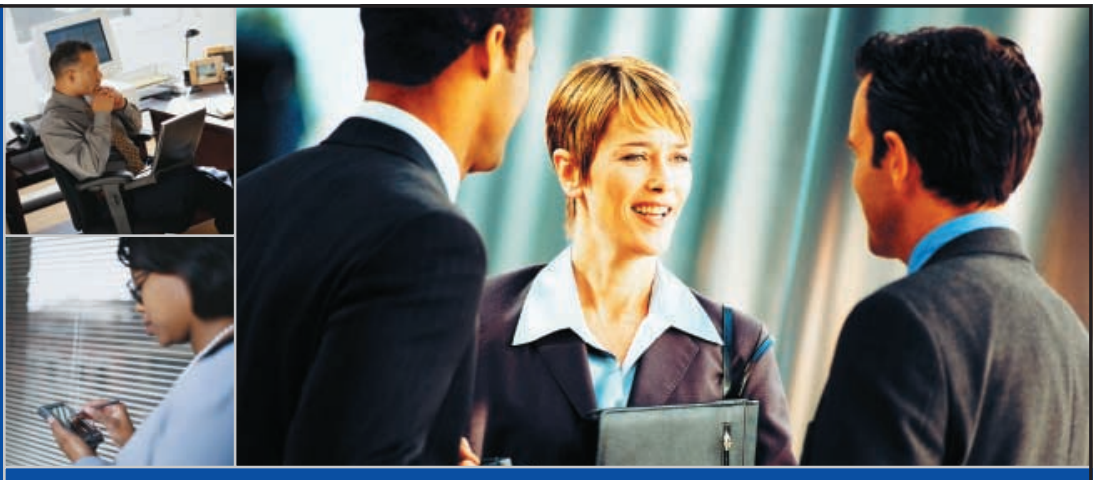

\section{for Physician Executives}

Helping physician executives and managers make informed decisions about information technology

September 17-18, 2004

Sheraton Centre Toronto

Toronto, Ontario

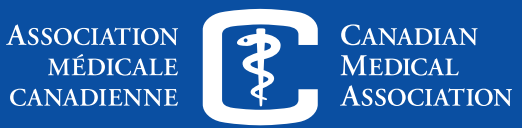

Learn from your peers and make valuable contacts at this highly interactive and professional workshop being offered by the Canadian Medical Association.

Led by Victoria-based information technology (IT) expert, Denis Protti, this workshop will focus on strategic IT decision-making and technology investment, and the important role of physician leaders in the success of IT health care initiatives.

For registration and program information please visit cma.ca/ITworkshop.htm or contact the CMA Member Service Centre at 1888 855-2555 or by email at cmamsc@cma.ca

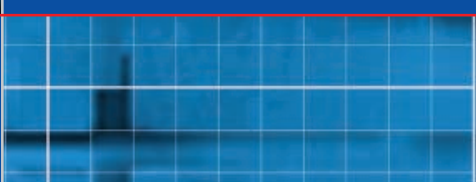

बme ce: 